\title{
RELIABILITY OF DIFFERENCE ANALOGUES TO PRESERVE STABILITY PROPERTIES OF STOCHASTIC VOLTERRA INTEGRO-DIFFERENTIAL EQUATIONS
}

\author{
LEONID E. SHAIKHET AND JASON A. ROBERTS
}

Received 2 August 2004; Revised 16 January 2005; Accepted 10 April 2005

We consider the reliability of some numerical methods in preserving the stability properties of the linear stochastic functional differential equation $d x(t)=\left(\alpha x(t)+\beta \int_{0}^{t} x(s) d s\right) d t$ $+\sigma x(t-\tau) d W(t)$, where $\alpha, \beta, \sigma, \tau \geq 0$ are real constants, and $W(t)$ is a standard Wiener process. The areas of the regions of asymptotic stability for the class of methods considered, indicated by the sufficient conditions for the discrete system, are shown to be equal in size to each other and we show that an upper bound can be put on the time-step parameter for the numerical method for which the system is asymptotically mean-square stable. We illustrate our results by means of numerical experiments and various stability diagrams. We examine the extent to which the continuous system can tolerate stochastic perturbations before losing its stability properties and we illustrate how one may accurately choose a numerical method to preserve the stability properties of the original problem in the numerical solution. Our numerical experiments also indicate that the quality of the sufficient conditions is very high.

Copyright (c) 2006 L. E. Shaikhet and J. A. Roberts. This is an open access article distributed under the Creative Commons Attribution License, which permits unrestricted use, distribution, and reproduction in any medium, provided the original work is properly cited.

\section{Introduction}

Volterra integro-differential equations arise in the modelling of hereditary systems (i.e., systems where the past influences the present) such as population growth, pollution, financial markets and mechanical systems (see, e.g., $[1,4]$ ). The long-term behaviour and stability of such systems is an important area for investigation. For example-will a population decline to dangerously low levels? Could a small change in the environmental conditions have drastic consequences on the long-term survival of the population? There is a growing body of works devoted to such investigations (see, e.g., [8, 25]). Analytical solutions to such problems are generally unavailable and numerical methods are adopted for obtaining approximate solutions. A large number of the numerical methods are developed from existing numerical methods for systems of ordinary differential

Hindawi Publishing Corporation

Advances in Difference Equations

Volume 2006, Article ID 73897, Pages 1-22

DOI 10.1155/ADE/2006/73897 
equations (see [24] for a discussion of some of these methods for ODEs). A natural question to ask is "do the numerical solutions preserve the stability properties of the exact solution?". We refer the reader to a number of works where the answers to such questions are investigated: $[2,3,6,7,9,28]$.

Many real-world phenomena are subject to random noise or perturbations (e.g., freak weather conditions may adversely affect the supports of a bridge, possibly changing the long-term integrity of the structure). It is a natural extension of the deterministic work carried out by ourselves and others to consider the stability of stochastic systems and of numerical solutions to such systems. We refer the readers to a number of texts which discuss the role of stochastic systems in mathematical modelling: [1, 15, 27]. In particular, stochastic integro-differential equations and its difference analogues are considered in $[5,11-14,26]$.

In this paper we consider the scalar linear test equation

$$
\begin{gathered}
d x(t)=\left(\alpha x(t)+\beta \int_{0}^{t} x(s) d s\right) d t+\sigma x(t-\tau) d W(t), \\
x(s)=\varphi_{0}(s), \quad s \in[-\tau, 0]
\end{gathered}
$$

where $\alpha, \beta, \sigma, \tau \geq 0$ are real constants, and $W(t)$ is a standard Wiener process. General theory of stochastic differential equations type of (1.1) was studied by Gikhman and Skorokhod [10].

The selected test equation (1.1) arises from the deterministic linear test equation of Brunner and Lambert [2] $\dot{x}(t)=\alpha x(t)+\beta \int_{0}^{t} x(s) d s$ by replacing the parameter $\alpha$ with its mean-value plus a stochastic perturbations type of the white noise $\alpha+\sigma \dot{W}(t)$. This leads to the stochastic differential equation $d x(t)=\left(\alpha x(t)+\beta \int_{0}^{t} x(s) d s\right) d t+\sigma x(t) d W(t)$. An addition of delay $\tau \geq 0$ in the stochastic term of this equation is a quite natural generalization and leads to (1.1). The delay $\tau$ does not have any influence on the obtained stability conditions but allows to demonstrate the construction of these conditions more completely. On the other hand even for $\tau=0$ the difference analogue of (1.1) is a difference equation with delay. So, an addition of delay $\tau$ does not lead to the essential complication of the text.

When considering the stability of a system we must decide on a suitable definition for stability. There are a number of definitions for the stability of stochastic systems. A common choice of definition amongst numerical analysts investigating stochastic differential equations is that of mean square stability and asymptotic mean square stability. We derive asymptotic mean square stability conditions for the linear test equation (1.1). An analogous approach is used to derive conditions for asymptotic mean square stability of a linear stochastic difference equation. It is shown that our choice of numerical methods are special cases of this particular difference equation, thereby allowing us to produce stability conditions for the numerical solutions to the original problem. Finally, we present some stability diagrams and numerical experiments to illustrate our results. 
The main conclusion of our investigation here can be formulated in the following way: if the trivial solution of the initial functional differential equation is asymptotically mean square stable then there exists a method and a step of discretization of this equation so that the trivial solution of the corresponding difference equation is asymptotically mean square stable too. Moreover, it is possible to find an upper bound for the step of discretization for which the corresponding discrete analogue preserves the properties of stability.

The conditions for asymptotic mean square stability are obtained here by virtue of Kolmanovskii and Shaikhet's general method of Lyapunov functionals construction ([1723, 29, 31-33]) which is applicable for both differential and difference equations, both for deterministic and stochastic systems with delay.

Let us remind ourselves of some definitions and statements which will be used.

Let $\{\Omega, \mathscr{F}, \mathbf{P}\}$ be a basic probability space with a family of $\sigma$-algebras $f_{t} \subset \mathscr{F}, t \geq 0$, and $H$ be a space of $f_{0}$-adapted functions $\varphi(s), s \leq 0$. Let $\mathbf{E}$ be the sign for expectation.

Consider a stochastic differential equation with aftereffect

$$
d x(t)=a\left(t, x_{t}\right) d t+b\left(t, x_{t}\right) d W(t), \quad x_{0}=\varphi_{0} \in H .
$$

Hence $W(t) \in \mathbb{R}^{m}$ is an $m$-dimensional Wiener process, the functionals $a(t, \varphi) \in \mathbb{R}^{n}$ and $b(t, \varphi) \in \mathbb{R}^{n \times m}$ are defined for $t \geq 0, \varphi \in H, a(t, 0)=0, b(t, 0)=0 . x_{t}(s)=x(t+s), s \leq 0$, is a trajectory of the process $x(s)$ for $s \leq t$.

Definition 1.1. The trivial solution of (1.3) is called

(i) mean square stable if for every $\epsilon>0$ there exists a $\delta=\delta(\epsilon)>0$ such that $\mathbb{E}|x(t)|^{2}<$ $\epsilon$ for all $t \geq 0$ if $\sup _{s \leq 0} \mathbb{E}|\varphi(s)|^{2}<\delta$;

(ii) asymptotically mean square stable if it is mean square stable and $\lim _{t \rightarrow \infty} \mathbf{E}|x(t)|^{2}=$ 0 for every initial function $\varphi \in H$.

Let $D$ be a space of functionals $V(t, \varphi)$, where $t \geq 0, \varphi \in H$, for which the function

$$
V_{\varphi}(t, x)=V\left(t, x_{t}\right)=V\left(t, x, x_{t}(s), s<0\right), \quad x=x(t), \quad \varphi=x_{t},
$$

has one continuous derivative with respect to $t$ and two continuous derivatives with respect to $x$. For each functional $V$ from $D$ the generator $L$ is defined by the formula

$$
L V(t, \varphi)=\frac{\partial}{\partial t} V_{\varphi}(t, x)+a^{\prime}(t, \varphi) \frac{\partial}{\partial x} V_{\varphi}(t, x)+\frac{1}{2} \operatorname{tr}\left[b^{\prime}(t, \varphi) \frac{\partial^{2}}{\partial x^{2}} V_{\varphi}(t, x) b(t, \varphi)\right],
$$

where the prime symbol ' denotes transpose.

Theorem $1.2([16,17])$. Let there exist a functional $V=V(t, \varphi) \in D$ such that

$$
\begin{gathered}
\mathbf{E} V\left(t, x_{t}\right) \geq c_{1} \mathbf{E}|x(t)|^{2}, \\
\mathbf{E} V\left(0, \varphi_{0}\right) \leq c_{2} \sup _{s \leq 0} \mathbf{E}\left|\varphi_{0}(s)\right|^{2}, \\
\mathbf{E} L V\left(t, x_{t}\right) \leq-c_{3} \mathbf{E}|x(t)|^{2},
\end{gathered}
$$

where $c_{i}>0, i=1,2,3$. Then the trivial solution of (1.3) is asymptotically mean square stable. 


\section{Reliability to preserve stability properties}

Let $\{\Omega, \mathscr{F}, \mathbf{P}\}$ be a basic probability space, $f_{i} \in \mathscr{F}_{\mathscr{F}}, i \in Z=\{0,1, \ldots\}$ be a sequence of $\sigma$-algebras, $\xi_{i} \in \mathbb{R}^{m}, i \in Z$ be $f_{i+1}$-adapted and mutually independent random variables. Suppose also that $\mathbf{E} \xi_{i}=0, \mathbf{E} \xi_{i} \xi_{i}^{\prime}=I$, where $I$ is an identity matrix.

Consider a stochastic difference equation

$$
x_{i+1}=a\left(i, x_{-m}, \ldots, x_{i}\right)+b\left(i, x_{-m}, \ldots, x_{i}\right) \xi_{i}, \quad i \in Z \text {. }
$$

Here $a \in \mathbb{R}^{n}, b \in \mathbb{R}^{n \times m}, a(i, 0, \ldots, 0)=0, b(i, 0, \ldots, 0)=0, x_{i}=\varphi_{i}, i \in[-m, 0]$.

Definition 1.3. The trivial solution of (1.7) is called:

(i) mean square stable if for every $\epsilon>0$ there exists $\delta=\delta(\epsilon)>0$ such that $\mathbf{E}\left|x_{i}\right|^{2}<\epsilon$, $i \in Z$, if $\sup _{i \in[-m, 0]} \mathbf{E}\left|\varphi_{i}\right|^{2}<\delta$;

(ii) asymptotically mean square stable if $\lim _{i \rightarrow \infty} \mathbf{E}\left|x_{i}\right|^{2}=0$ for every initial function $\varphi_{i}$. Theorem 1.4 [20]. Let there exist a nonnegative functional $V_{i}=V\left(i, x_{-m}, \ldots, x_{i}\right)$, which satisfies the conditions

$$
\begin{gathered}
\mathrm{E} V\left(0, x_{-m}, \ldots, x_{0}\right) \leq c_{1} \sup _{i \leq 0} \mathbf{E}\left|\varphi_{i}\right|^{2}, \\
\mathbf{E} \Delta V_{i} \leq-c_{2} \mathbf{E}\left|x_{i}\right|^{2}, \quad i \in Z,
\end{gathered}
$$

where $c_{1}>0, c_{2}>0, \Delta V_{i}=V_{i+1}-V_{i}$. Then the trivial solution of (1.7) is asymptotically mean square stable.

\section{A linear stochastic Volterra integro-differential equation}

Consider (1.1). It is well known [16] that for $\beta=0$ the inequality

$$
2 \alpha+\sigma^{2}<0
$$

is the necessary and sufficient condition for the asymptotic mean square stability of the trivial solution of (1.1).

If $\sigma=0$ then (1.1) reduces to the Brunner and Lambert test equation [2] and also takes the differential form

$$
\ddot{x}(t)=\alpha \dot{x}(t)+\beta x(t) .
$$

In this case the inequalities

$$
\alpha<0, \quad \beta<0,
$$

are the necessary and sufficient condition for asymptotic stability of the trivial solution of (1.1).

We proceed in the following way to obtain asymptotic mean square stability conditions for the trivial solution of (1.1) via Lyapunov's second method. Following conditions (2.1), 
(2.3) we will suppose that the conditions

$$
2 \alpha+\sigma^{2}<0, \quad \beta<0,
$$

hold.

We transform (1.1) in the following way. Let

$$
y_{1}(t)=\int_{0}^{t} x(s) d s, \quad y_{2}(t)=x(t) .
$$

Then (1.1) is transformed into the system of equations

$$
\begin{gathered}
d y_{1}(t)=y_{2}(t) d t \\
d y_{2}(t)=\left(\beta y_{1}(t)+\alpha y_{2}(t)\right) d t+\sigma y_{2}(t-\tau) d W(t)
\end{gathered}
$$

or in the matrix form

$$
d y(t)=A y(t) d t+B y(t-\tau) d W(t)
$$

where

$$
y=\left(\begin{array}{l}
y_{1} \\
y_{2}
\end{array}\right), \quad A=\left(\begin{array}{ll}
0 & 1 \\
\beta & \alpha
\end{array}\right), \quad B=\left(\begin{array}{ll}
0 & 0 \\
0 & \sigma
\end{array}\right) .
$$

Following the general method of Lyapunov functionals construction $[17,18]$ we will construct a Lyapunov functional for (2.7) in the form $V=V_{1}+V_{2}$, where the main part $V_{1}$ of the functional $V$ must be chosen as a Lyapunov function for some auxiliary differential equation without delay (in this case it is $(2.7)$ with $B=0$ ). Let us choose $V_{1}$ in the form $V_{1}=y^{\prime}(t) P y(t)$ where $P=\left(\begin{array}{l}p_{11} p_{12} \\ p_{12} p_{22}\end{array}\right)$ is a positive definite matrix. Calculating for $(2.7)$ the generator $L$ we obtain

$$
\mathbf{E} L V_{1}=\mathbf{E} y^{\prime}(t)\left(P A+A^{\prime} P\right) y(t)+\mathbf{E} y^{\prime}(t-\tau) B^{\prime} P B y(t-\tau)
$$

Let us choose the additional functional $V_{2}$ in the form

$$
V_{2}=\int_{t-\tau}^{t} y^{\prime}(s) B^{\prime} P B y(s) d s .
$$

Then

$$
\mathbf{E} L V_{2}=\mathbf{E} y^{\prime}(t) B^{\prime} P B y(t)-\mathbf{E} y^{\prime}(t-\tau) B^{\prime} P B y(t-\tau)
$$

and from (2.9), (2.11) it follows for the functional $V=V_{1}+V_{2}$ that

$$
\mathbf{E} L V=\mathbf{E} y^{\prime}(t)\left(P A+A^{\prime} P+B^{\prime} P B\right) y(t)
$$

Suppose that the matrix $P$ is a positive definite solution of the matrix equation

$$
P A+A^{\prime} P+B^{\prime} P B=-I,
$$


where $I$ is the identity matrix. Matrix equation (2.13) is equivalent to the system of the equations

$$
\begin{gathered}
2 \beta p_{12}=-1, \\
p_{11}+\alpha p_{12}+\beta p_{22}=0, \\
2 p_{12}+\left(2 \alpha+\sigma^{2}\right) p_{22}=-1,
\end{gathered}
$$

with the solution

$$
p_{11}=\frac{\alpha}{2 \beta}-\frac{1-\beta}{2 \alpha+\sigma^{2}}, \quad p_{12}=-\frac{1}{2 \beta}, \quad p_{22}=\frac{1-\beta}{\beta\left(2 \alpha+\sigma^{2}\right)} .
$$

It is easy to check by conditions (2.4) that $p_{11}>0, p_{22}>0$ and $p_{11} p_{22}>p_{12}^{2}$. Therefore the matrix $P$ with elements (2.15) is positive definite, as required. From here and (2.12), (2.13) it follows that there exists a positive definite functional $V$, for which $L V=$ $-|y(t)|^{2}$. Recalling our originally supposed conditions, (2.1) with $\beta=0,(2.4)$, and using [16] we can now state the following result.

Theorem 2.1. The system of inequalities

$$
2 \alpha+\sigma^{2}<0, \quad \beta \leq 0,
$$

is the necessary and sufficient condition for asymptotic mean square stability of the trivial solution of (1.1).

\section{Stability of difference analogues to the integro-differential equation}

Let $\{\Omega, \mathscr{F}, P\}$ be a basic probability space, $f_{i} \in \mathscr{F}, i \in Z=\{0,1, \ldots\}$ be a sequence of $\sigma$ algebras and $\mathbf{E}$ be the sign for expectation. If we quantify equation (1.1) using a numerical method based on the Euler-Maruyama scheme for the stochastic differential equation part and a $\theta$ method to approximate the integral with a quadrature, then we obtain a family of numerical methods of the form

$$
\begin{gathered}
x_{1}=(a+b) x_{0}+\sigma_{0} x_{-m} \xi_{0}, \\
x_{2}=a x_{1}+b\left(\theta x_{0}+(1-\theta) x_{1}\right)+\sigma_{0} x_{1-m} \xi_{1}, \\
x_{i+1}=a x_{i}+b\left(\theta x_{0}+\sum_{j=1}^{i-1} x_{j}+(1-\theta) x_{i}\right)+\sigma_{0} x_{i-m} \xi_{i}, \quad i \geq 2, \\
a=1+\alpha h, \quad b=\beta h^{2}, \sigma_{0}=\sigma h^{1 / 2},
\end{gathered}
$$

where $\theta \in[0,1], \tau=m h, h=t_{i+1}-t_{i}$ is a step of quantization, $\xi_{i}=h^{-1 / 2}\left(W\left(t_{i+1}\right)-\right.$ $\left.W\left(t_{i}\right)\right), i \in Z$, are $f_{i+1}$-adapted and mutually independent random variables such that $\mathbf{E} \xi_{i}=0, \mathbf{E} \xi_{i}^{2}=1$.

Note that if $b=0$ then the inequality

$$
a^{2}+\sigma_{0}^{2}<1
$$


is the necessary and sufficient condition for asymptotic mean square stability of the trivial solution of (3.1) [29].

Suppose that $b \neq 0$. We transform (3.1) for $i \geq 2$ in the following way:

$$
\begin{aligned}
x_{i+1}= & (a+b(1-\theta)) x_{i}+b x_{i-1}+b\left(\theta x_{0}+\sum_{j=1}^{i-2} x_{j}\right)+\sigma_{0} x_{i-m} \xi_{i} \\
= & (a+b(1-\theta)) x_{i}+b x_{i-1}+\sigma_{0} x_{i-m} \xi_{i}+x_{i} \\
& -(a+b(1-\theta)) x_{i-1}-\sigma_{0} x_{i-1-m} \xi_{i-1} \\
= & (a+b(1-\theta)+1) x_{i}+(b \theta-a) x_{i-1}+\sigma_{0} x_{i-m} \xi_{i}-\sigma_{0} x_{i-1-m} \xi_{i-1} .
\end{aligned}
$$

As a result we obtain (3.1) in the form

$$
x_{i+1}=A x_{i}+B x_{i-1}+\sigma_{1} x_{i-m} \xi_{i}+\sigma_{2} x_{i-1-m} \xi_{i-1}, \quad i \geq 2,
$$

where

$$
A=a+b(1-\theta)+1, \quad B=b \theta-a, \quad \sigma_{1}=\sigma_{0}, \quad \sigma_{2}=-\sigma_{0} .
$$

It is known [29] that for $\sigma_{2}=0$ the necessary and sufficient condition for asymptotic mean square stability of the trivial solution of (3.4) is

$$
\begin{gathered}
|A|<1-B, \quad|B|<1, \\
\sigma_{1}^{2}<\frac{1+B}{1-B}\left((1-B)^{2}-A^{2}\right) .
\end{gathered}
$$

We now obtain a sufficient condition for asymptotic mean square stability of the trivial solution of (3.4) for arbitrary $\sigma_{1}$ and $\sigma_{2}$. Let

$$
x(i)=\left(\begin{array}{c}
x_{i-1} \\
x_{i}
\end{array}\right), \quad A_{1}=\left(\begin{array}{cc}
0 & 1 \\
B & A
\end{array}\right), \quad B_{k}=\left(\begin{array}{c}
0 \\
\sigma_{k}
\end{array}\right), \quad k=1,2 .
$$

Then (3.4) takes the following matrix form:

$$
x(i+1)=A_{1} x(i)+B_{1} x_{i-m} \xi_{i}+B_{2} x_{i-1-m} \xi_{i-1} .
$$

Using the general method of Lyapunov functionals construction [20] let us construct a Lyapunov functional $V_{i}$ for (3.9). This method consists of four steps. On the first step of the method we have to consider some simple auxiliary difference equation. In the case of (3.9) the auxiliary difference equation is the equation without delay $x(i+1)=A_{1} x(i)$ (i.e., (3.9) with $B_{1}=B_{2}=0$ ). On the second step we have to construct a Lyapunov function $v_{i}$ for this auxiliary difference equation. Let

$$
v_{i}=x^{\prime}(i) D x(i), \quad D=\left(\begin{array}{ll}
d_{11} & d_{12} \\
d_{12} & d_{22}
\end{array}\right)
$$


8 Reliability to preserve stability properties

and suppose that the matrix $D$ is a positive semi-definite solution of the matrix equation

$$
A_{1}^{\prime} D A_{1}-D=-U, \quad U=\left(\begin{array}{ll}
0 & 0 \\
0 & 1
\end{array}\right),
$$

with $d_{22}>0$. It is easy to check that the function $v_{i}$ is a Lyapunov function for the equation $x(i+1)=A_{1} x(i)$ since $\Delta v_{i}=-x_{i}^{2}$. On the third step we will construct the functional $V_{i}$ for (3.9) in the form $V_{i}=V_{1 i}+V_{2 i}$, where the main part $V_{1 i}=v_{i}$ and the additional part $V_{2 i}$ will be chosen below. Calculating $\mathbf{E} \Delta V_{1 i}=\mathbf{E}\left(V_{1, i+1}-V_{1 i}\right)$, by virtue of (3.10), (3.9) we obtain

$$
\begin{aligned}
\mathbf{E} \Delta V_{1 i}=\mathbf{E}\left(x^{\prime}(i+1) D x(i+1)-x^{\prime}(i) D x(i)\right) \\
=\mathbf{E}\left(\left(A_{1} x(i)+B_{1} x_{i-m} \xi_{i}+B_{2} x_{i-1-m} \xi_{i-1}\right)^{\prime}\right. \\
\left.\quad \times D\left(A_{1} x(i)+B_{1} x_{i-m} \xi_{i}+B_{2} x_{i-1-m} \xi_{i-1}\right)-x^{\prime}(i) D x(i)\right) \\
=\mathbf{E}\left(x^{\prime}(i)\left(A_{1}^{\prime} D A_{1}-D\right) x(i)+B_{1}^{\prime} D B_{1} x_{i-m}^{2} \xi_{i}^{2}\right. \\
\quad+B_{2}^{\prime} D B_{2} x_{i-1-m}^{2} \xi_{i-1}^{2}+2 B_{1}^{\prime} D A_{1} x(i) x_{i-m} \xi_{i} \\
\left.\quad+2 B_{2}^{\prime} D A_{1} x(i) x_{i-1-m} \xi_{i-1}+2 B_{1}^{\prime} D B_{2} x_{i-m} x_{i-1-m} \xi_{i} \xi_{i-1}\right) .
\end{aligned}
$$

From (3.11) it follows that

$$
\mathbf{E} x^{\prime}(i)\left(A_{1}^{\prime} D A_{1}-D\right) x(i)=-\mathbf{E} x_{i}^{2} .
$$

From (3.8), (3.10) and the properties of $\xi_{i}$, we obtain

$$
\begin{gathered}
\mathbf{E} x_{i-m}^{2} \xi_{i}^{2}=\mathbf{E} x_{i-m}^{2}, \\
\mathbf{E} x(i) x_{i-m} \xi_{i}=0, \\
\mathbf{E} x_{i-m} x_{i-1-m} \xi_{i} \xi_{i-1}=0, \\
B_{2}^{\prime} D A_{1}=\left(\sigma_{2} B d_{22}, \sigma_{2}\left(d_{12}+A d_{22}\right)\right), \\
B_{k}^{\prime} D B_{k}=\sigma_{k}^{2} d_{22}, \quad k=1,2, \\
\mathbf{E} x(i) x_{i-1-m} \xi_{i-1}=\left(0, \mathbf{E} x_{i} x_{i-1-m} \xi_{i-1}\right)^{\prime} .
\end{gathered}
$$

Using (3.4), we have

$$
\begin{aligned}
\mathbf{E} x_{i} x_{i-1-m} \xi_{i-1} & =\mathbf{E}\left(A x_{i-1}+B x_{i-2}+\sigma_{1} x_{i-1-m} \xi_{i-1}+\sigma_{2} x_{i-2-m} \xi_{i-2}\right) x_{i-1-m} \xi_{i-1} \\
& =\sigma_{1} \mathbf{E} x_{i-1-m}^{2} .
\end{aligned}
$$

From (3.12) to (3.15) we obtain

$$
\mathbf{E} \Delta V_{1 i}=-\mathbf{E} x_{i}^{2}+\sigma_{1}^{2} d_{22} \mathbf{E} x_{i-m}^{2}+\left(\sigma_{2}^{2} d_{22}+2 \sigma_{1} \sigma_{2}\left(d_{12}+A d_{22}\right)\right) \mathbf{E} x_{i-1-m}^{2} \text {. }
$$

Using (3.8), (3.10) we have

$$
A_{1}^{\prime} D A_{1}=\left(\begin{array}{cc}
B^{2} d_{22} & B\left(d_{12}+A d_{22}\right) \\
B\left(d_{12}+A d_{22}\right) & d_{11}+2 A d_{12}+A^{2} d_{22}
\end{array}\right) .
$$


Using (3.17) one can transform matrix equation (3.11) into the system of equations

$$
\begin{gathered}
B^{2} d_{22}-d_{11}=0, \\
B\left(d_{12}+A d_{22}\right)-d_{12}=0, \\
d_{11}+2 A d_{12}+A^{2} d_{22}-d_{22}=-1 .
\end{gathered}
$$

The solution of system (3.18) has the form

$$
\begin{gathered}
d_{11}=B^{2} d_{22}, \\
d_{12}=\frac{A B}{1-B} d_{22}, \\
d_{22}=\left(\frac{1+B}{1-B}\left((1-B)^{2}-A^{2}\right)\right)^{-1}
\end{gathered}
$$

Note that $d_{22}>0$ if and only if condition (3.6) holds. Substituting (3.19) into (3.16), we have

$$
\mathbf{E} \Delta V_{1 i}=-\mathbf{E} x_{i}^{2}+\sigma_{1}^{2} d_{22} \mathbf{E} x_{i-m}^{2}+\gamma d_{22} \mathbf{E} x_{i-1-m}^{2}
$$

where

$$
\gamma=\sigma_{2}^{2}+2 \sigma_{1} \sigma_{2} \frac{A}{1-B}
$$

Letting $\gamma_{0}=\max (\gamma, 0)$ we can at last (the fourth step of the method) by some standard way choose the additional functional

$$
V_{2 i}=d_{22}\left(\left(\sigma_{1}^{2}+\gamma_{0}\right) \sum_{j=1}^{m} x_{i-j}^{2}+\gamma_{0} x_{i-1-m}^{2}\right) .
$$

It follows that

$$
\begin{aligned}
\Delta V_{2 i} & =d_{22}\left(\left(\sigma_{1}^{2}+\gamma_{0}\right)\left(\sum_{j=1}^{m} x_{i+1-j}^{2}-\sum_{j=1}^{m} x_{i-j}^{2}\right)+\gamma_{0}\left(x_{i-m}^{2}-x_{i-1-m}^{2}\right)\right) \\
& =d_{22}\left(\left(\sigma_{1}^{2}+\gamma_{0}\right)\left(x_{i}^{2}-x_{i-m}^{2}\right)+\gamma_{0}\left(x_{i-m}^{2}-x_{i-1-m}^{2}\right)\right) \\
& =d_{22}\left(\left(\sigma_{1}^{2}+\gamma_{0}\right) x_{i}^{2}-\sigma_{1}^{2} x_{i-m}^{2}-\gamma_{0} x_{i-1-m}^{2}\right) .
\end{aligned}
$$

So, using (3.16), (3.23) for the functional $V_{i}=V_{1 i}+V_{2 i}$ we have

$$
\mathbf{E} \Delta V_{i}=-\left(1-d_{22}\left(\sigma_{1}^{2}+\gamma_{0}\right)\right) \mathbf{E} x_{i}^{2}+d_{22}\left(\gamma-\gamma_{0}\right) \mathbf{E} x_{i-1-m}^{2} .
$$

If $\gamma \geq 0$ then $\gamma_{0}=\gamma$ and, using (3.21), we obtain

$$
\mathbf{E} \Delta V_{i}=-\left(1-d_{22}\left(\sigma_{1}^{2}+2 \sigma_{1} \sigma_{2} \frac{A}{1-B}+\sigma_{2}^{2}\right)\right) \mathbf{E} x_{i}^{2} .
$$


From here and representation (3.18) for $d_{22}$ it follows [29] that if $\gamma \geq 0$ then the inequality

$$
\sigma_{1}^{2}+2 \sigma_{1} \sigma_{2} \frac{A}{1-B}+\sigma_{2}^{2}<\frac{1+B}{1-B}\left((1-B)^{2}-A^{2}\right)
$$

is the necessary and sufficient condition for asymptotic mean square stability of the trivial solution of (3.4).

Consider now the situation if $\gamma<0$. In this case $\gamma_{0}=0$ and (3.24) takes the form

$$
\mathbf{E} \Delta V_{i}=-\left(1-\sigma_{1}^{2} d_{22}\right) \mathbf{E} x_{i}^{2}+\gamma d_{22} \mathbf{E} x_{i-1-m}^{2} .
$$

So, if $\gamma<0$ then the inequality $\sigma_{1}^{2} d_{22}<1$ is a sufficient condition for asymptotic mean square stability of the trivial solution of (3.4). Let us suppose that $\gamma<0$ and $\sigma_{1}^{2} d_{22} \geq 1$. Summing (3.27) from $i=0$ to $i=n$, we have

$$
\mathbf{E} V_{n+1}-\mathbf{E} V_{0}=-\left(1-\sigma_{1}^{2} d_{22}\right) \sum_{i=0}^{n} \mathbf{E} x_{i}^{2}+\gamma d_{22}\left(\sum_{i=0}^{n-1-m} \mathbf{E} x_{i}^{2}+\sum_{i=-1-m}^{-1} \mathbf{E} x_{i}^{2}\right) \text {. }
$$

From here, using $V_{n+1} \geq 0$ and $\gamma<0$, we obtain

$$
\left(1-\sigma_{1}^{2} d_{22}\right) \sum_{i=0}^{n} \mathbf{E} x_{i}^{2}-\gamma d_{22} \sum_{i=0}^{n-1-m} \mathbf{E} x_{i}^{2} \leq \mathbf{E} V_{0}
$$

or

$$
\left(1-d_{22}\left(\sigma_{1}^{2}+\gamma\right)\right) \sum_{i=0}^{n} \mathbf{E} x_{i}^{2} \leq \mathbf{E} V_{0}+|\gamma| d_{22} \sum_{i=n-m}^{n} \mathbf{E} x_{i}^{2} .
$$

Note that by virtue of (3.6) we have

$$
\sigma_{1}^{2}+\gamma=\sigma_{1}^{2}+2 \sigma_{1} \sigma_{2} \frac{A}{1-B}+\sigma_{2}^{2}>\sigma_{1}^{2}-2\left|\sigma_{1} \sigma_{2}\right|+\sigma_{2}^{2}=\left(\left|\sigma_{1}\right|-\left|\sigma_{2}\right|\right)^{2} \geq 0 .
$$

Therefore, by condition (3.26), that is equivalent to $d_{22}\left(\sigma_{1}^{2}+\gamma\right)<1$, each mean square bounded solution of (3.4), that is, $\mathbf{E} x_{i}^{2} \leq C$, satisfies the condition $\lim _{i \rightarrow \infty} \mathbf{E} x_{i}^{2}=0$.

So by condition (3.26) the mean square bounded solution of (3.4) is asymptotically mean square trivial, that is, $\lim _{i \rightarrow \infty} \mathbf{E} x_{i}^{2}=0$. Note also that for $\sigma_{2}=0$ condition (3.26) coincides with (3.1).

Using (3.5), (3.6), we rewrite condition (3.26) in terms of the parameters of (3.1):

$$
\begin{gathered}
\sigma_{0}^{2}<(1-a+b \theta)\left(1+a-b\left(\theta-\frac{1}{2}\right)\right), \\
b\left(\theta-\frac{1}{2}\right)-1<a<b \theta+1, \quad-4<b<0 .
\end{gathered}
$$

If $b \rightarrow 0$ then condition (3.32) takes form (3.2). Conditions (3.32), (3.2) can also be written in the form

$$
\left(a-\left(\theta-\frac{1}{4}\right) b\right)^{2}+\sigma_{0}^{2}<\left(1+\frac{b}{4}\right)^{2}
$$




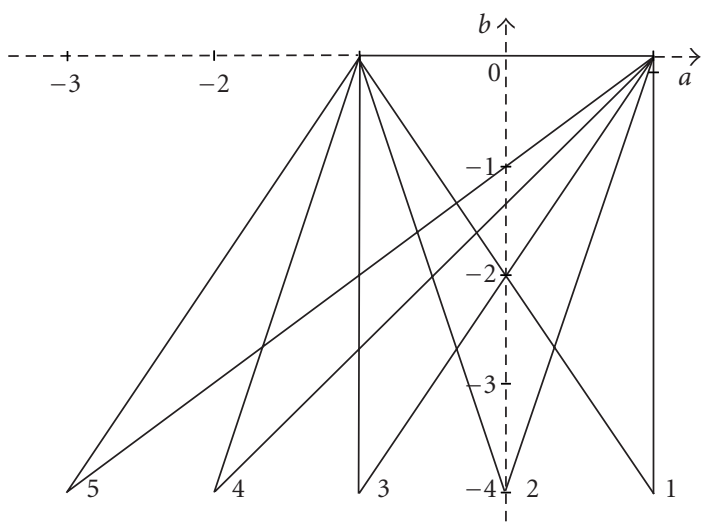

Figure 3.1. Stability diagram, $\sigma_{0}=0$, differing $\theta$ values.

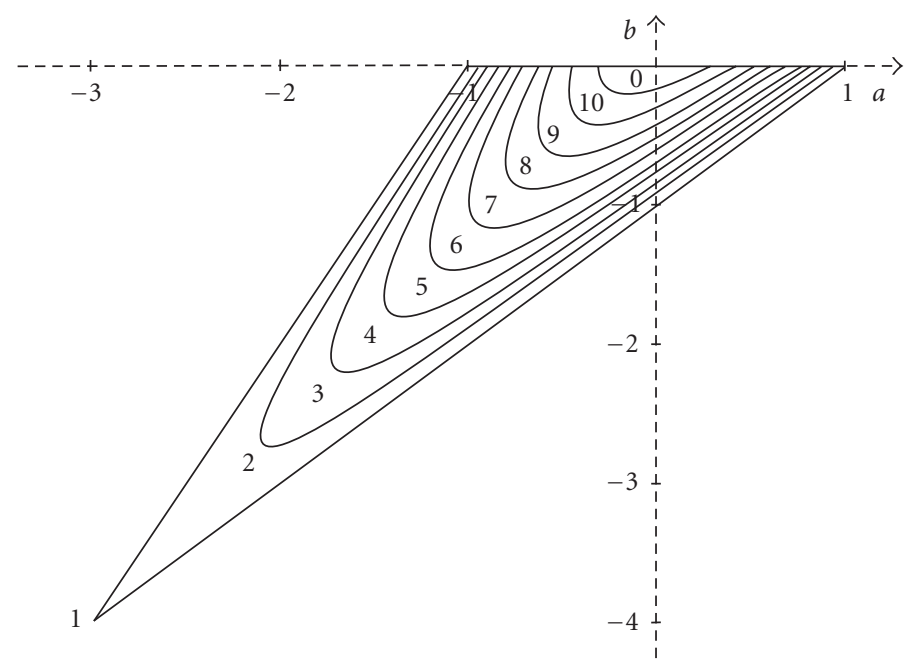

Figure 3.2. Stability diagram, $\theta=1$, differing $\sigma_{0}^{2}$ values.

or

$\left(\theta-\frac{1}{4}\right) b-\sqrt{\left(1+\frac{b}{4}\right)^{2}-\sigma_{0}^{2}}<a<\left(\theta-\frac{1}{4}\right) b+\sqrt{\left(1+\frac{b}{4}\right)^{2}-\sigma_{0}^{2}}, \quad-4\left(1-\left|\sigma_{0}\right|\right)<b \leq 0$.

Stability regions, obtained by virtue of condition (3.34) for $\sigma_{0}=0$ and different values of $\theta$ are shown in Figure 3.1 with the following key: (1) $\theta=0$, (2) $\theta=0.25$, (3) $\theta=0.5$, (4) $\theta=0.75$, (5) $\theta=1$. 


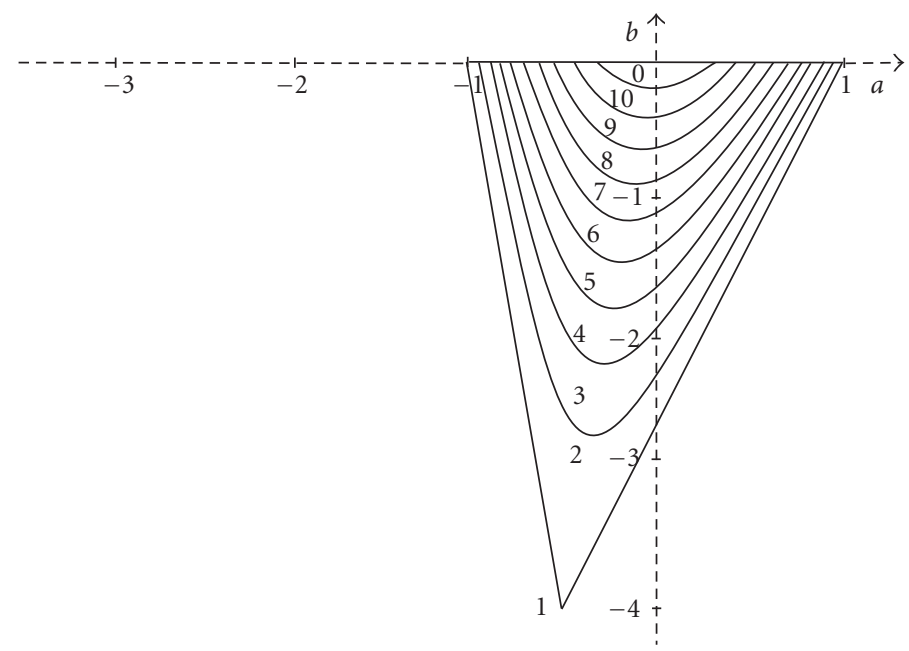

Figure 3.3. Stability diagram, $\theta=0.375$, differing $\sigma_{0}^{2}$ values.

Stability regions, obtained by virtue of condition (3.34) for $\theta=1$ and different values of $\sigma_{0}^{2}$ are shown in Figure 3.2 with the following key: (1) $\sigma_{0}^{2}=0$, (2) $\sigma_{0}^{2}=0.1$, (3) $\sigma_{0}^{2}=$ 0.2 , (4) $\sigma_{0}^{2}=0.3$, (5) $\sigma_{0}^{2}=0.4,(6) \sigma_{0}^{2}=0.5$, (7) $\sigma_{0}^{2}=0.6$, (8) $\sigma_{0}^{2}=0.7$, (9) $\sigma_{0}^{2}=0.8,(10)$ $\sigma_{0}^{2}=0.9$. Figure 3.3 uses the same key as Figure 3.2 and is for $\theta=0.375$.

Remark 3.1. Note that the stability region, given by condition (3.34) depends on $\theta$ and $\sigma_{0}$, but the area $S$ of this stability region depends on $\sigma_{0}$ only and does not depend on $\theta$, that is, $S=S\left(\sigma_{0}\right)$. It is easy to see that

$$
S\left(\sigma_{0}\right)=2 \int_{-4\left(1-\left|\sigma_{0}\right|\right)}^{0} \sqrt{\left(1+\frac{b}{4}\right)^{2}-\sigma_{0}^{2}} d b .
$$

Putting $t=x+\sqrt{x^{2}-\sigma_{0}^{2}}, x=1+b / 4$, one can show that

$$
S\left(\sigma_{0}\right)=4\left(\sqrt{1-\sigma_{0}^{2}}-\sigma_{0}^{2} \ln \left(\frac{1+\sqrt{1-\sigma_{0}^{2}}}{\left|\sigma_{0}\right|}\right)\right) .
$$

In particular, $S(0)=4, S(0.5)=2 \sqrt{3}-\ln (2+\sqrt{3})>2, S(1)=0$.

Stability condition (3.34) in the terms of initial equation (1.1) takes the form

$$
\begin{aligned}
& \frac{1}{h}\left(-1+\left(\theta-\frac{1}{4}\right) \beta h^{2}-\sqrt{\left(1+\frac{1}{4} \beta h^{2}\right)^{2}-\sigma^{2} h}\right)<\alpha \\
& \quad<\frac{1}{h}\left(-1+\left(\theta-\frac{1}{4}\right) \beta h^{2}+\sqrt{\left.\left(1+\frac{1}{4} \beta h^{2}\right)^{2}-\sigma^{2} h\right)}, \quad-4\left(1-|\sigma| h^{1 / 2}\right)<\beta h^{2} \leq 0 .\right.
\end{aligned}
$$




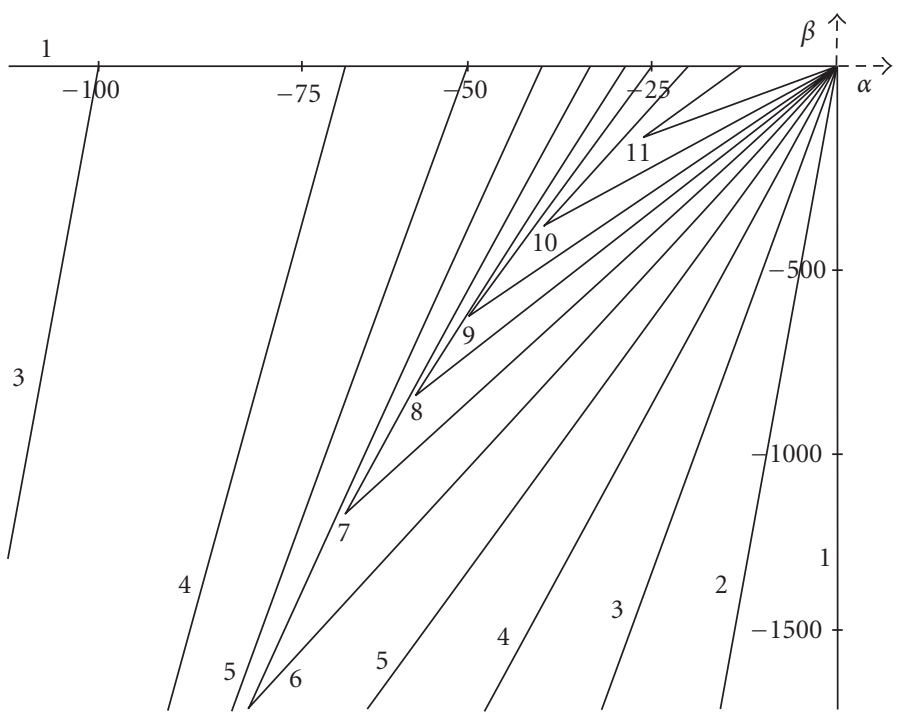

Figure 3.4. Stability diagram, $\theta=1, \sigma^{2}=0$, differing $h$ values.

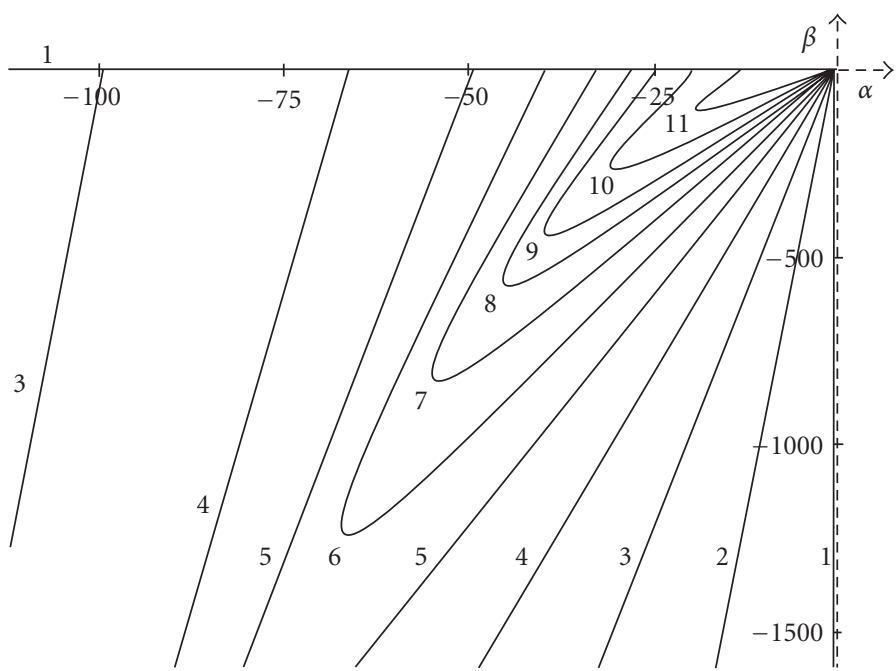

Figure 3.5. Stability diagram, $\theta=1, \sigma^{2}=1$, differing $h$ values.

The stability regions in the $(\alpha, \beta)$ space, obtained by condition (3.37) for $\theta=1, \sigma^{2}=0$ are shown in Figure 3.4 for different values of the step size $h$ of the numerical method, using the following key: (1) $h=0$, (2) $h=0.01$, (3) $h=0.02$, (4) $h=0.03$, (5) $h=0.04$, (6) $h=0.05$, (7) $h=0.06$, (8) $h=0.07$, (9) $h=0.08$, (10) $h=0.1$, (11) $h=0.15$. Figures 3.5 and 3.6 show similar pictures with $\theta=1$ and $h$ as indicated above but with $\sigma^{2}=1$ and $\sigma^{2}=3$ respectively. 


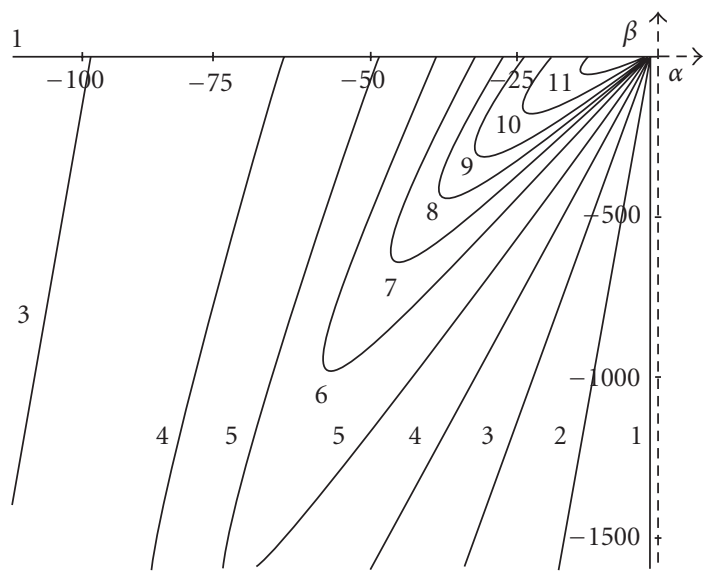

Figure 3.6. Stability diagram, $\theta=1, \sigma^{2}=3$, differing $h$ values.

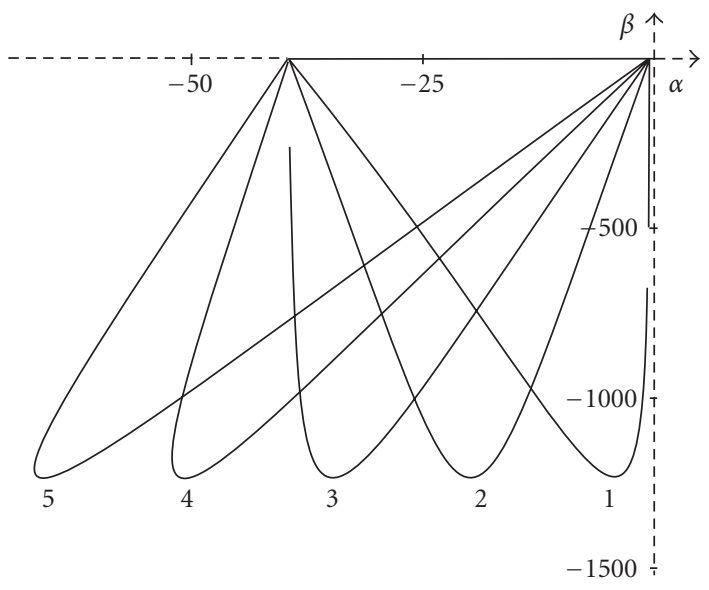

Figure 3.7. Stability diagram, $\sigma^{2}=1, h=0.05$, differing $\theta$ values.

Figure 3.7 illustrates the stability region in the $(\alpha, \beta)$ space for $\sigma^{2}=1, h=0.05$ and different values $\theta$ (i.e., different numerical schemes) according to the following key: (1) $\theta=0$, (2) $\theta=0.25$, (3) $\theta=0.5$, (4) $\theta=0.75$, (5) $\theta=1$.

If we calculate the infimum with respect to $\theta$ in the left-hand part and the supremum in the right-hand part of inequalities (3.37) we obtain

$$
\begin{aligned}
& \frac{1}{h}(-\left.1+\frac{3}{4} \beta h^{2}-\sqrt{\left(1+\frac{1}{4} \beta h^{2}\right)^{2}-\sigma^{2} h}\right)<\alpha \\
& \quad<\frac{1}{h}\left(-1-\frac{1}{4} \beta h^{2}+\sqrt{\left.\left(1+\frac{1}{4} \beta h^{2}\right)^{2}-\sigma^{2} h\right)}, \quad-4\left(1-|\sigma| h^{1 / 2}\right)<\beta h^{2} \leq 0 .\right.
\end{aligned}
$$




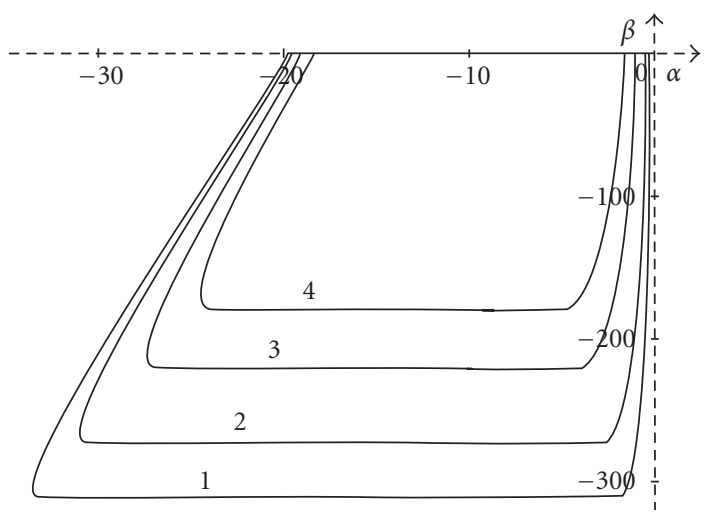

Figure 3.8. Stability diagram, $h=0.1$, differing $\sigma^{2}$ values.

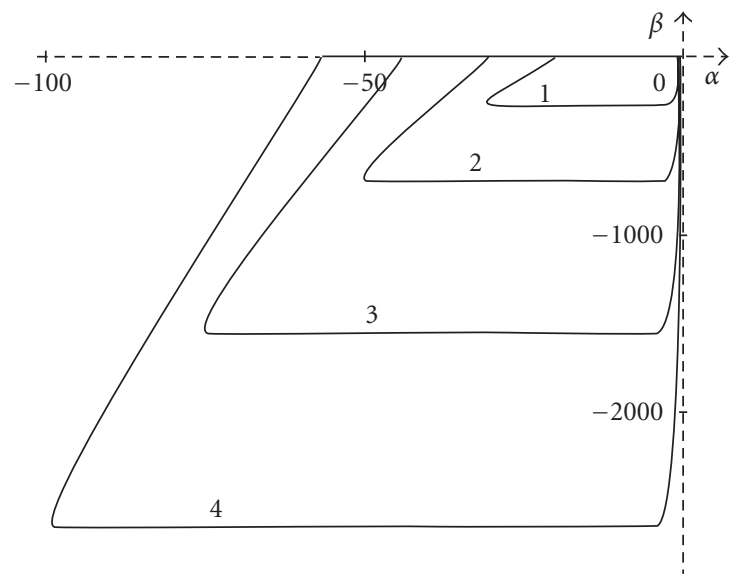

Figure 3.9. Stability diagram, $\sigma^{2}=1$, differing $h$ values.

It is easy to check that if $h \rightarrow 0$ then condition (3.38) coincides with condition (2.16). It leads to the following useful statement.

THEOREm 3.2. If $\alpha, \beta$ and $\sigma$ satisfy condition (2.16) then there exists a small enough $h$ such that condition (3.38) holds too. And if $\alpha, \beta, \sigma$ and $h$ satisfy condition (3.38) then there exists $a \theta \in[0,1]$ such that condition (3.37) holds too and therefore the trivial solution of (3.1) is asymptotically mean square stable.

The stability regions obtained by condition (3.38) for $h=0.1$ and different values of $\sigma$ are shown in Figure 3.8, according to the following key: (1) $\sigma^{2}=0.5$, (2) $\sigma^{2}=1$, (3) $\sigma^{2}=2$, (4) $\sigma^{2}=3$. Figure 3.9 shows a similar picture for $\sigma^{2}=1$ and different values of $h$ : (1) $h=0.1$, (2) $h=0.065$, (3) $h=0.045$, (4) $h=0.035$. 


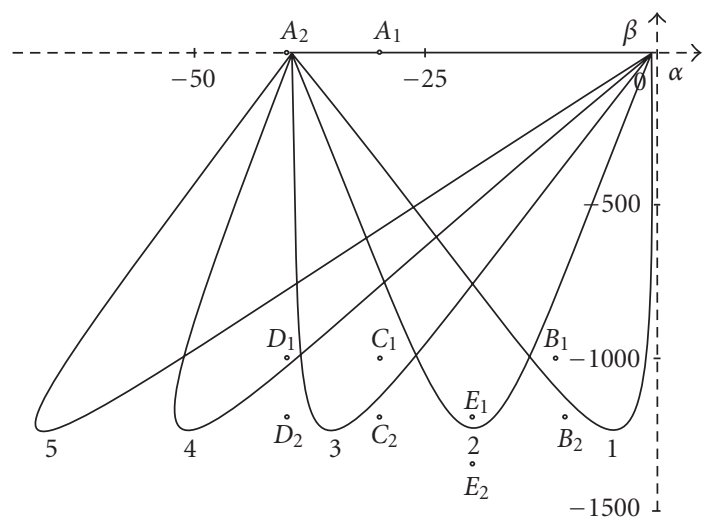

Figure 4.1. Stability diagram, $\sigma^{2}=1, h=0.05$, differing $\theta$ values.

\section{Upper bound for the step of discretization}

From condition (3.37) it follows that $f(h)>0$ where

$$
f(h):=\theta\left(\theta-\frac{1}{2}\right) \beta^{2} h^{3}-\left(2 \theta-\frac{1}{2}\right) \alpha \beta h^{2}+\left(\alpha^{2}-2 \beta \theta\right) h+2 \alpha+\sigma^{2} .
$$

Using the representation (4.1) consider different possible cases for determining an upper bound for the step of discretization.

4.1. Case $\beta=0$. Let $\beta=0$. From (4.1), (2.16) we obtain $f(h)=\alpha^{2} h+2 \alpha+\sigma^{2}<0$ for $h \in\left[0, h_{1}\right)$, where

$$
h_{1}=-\frac{2 \alpha+\sigma^{2}}{\alpha^{2}}>0
$$

For example, if $\alpha=-30, \beta=0, \sigma^{2}=1$ then $h_{1} \approx 0.0656$. Changing $\alpha$ to $\alpha=-40$, we obtain $h_{1} \approx 0.0494$. On Figure 4.1 which coincides with Figure $3.7\left(\sigma^{2}=1, h=0.05\right)$ the points $A_{1}(-30,0)$ and $A_{2}(-40,0)$ are shown. One can see that the point $A_{1}$ belongs to the stability region but the point $A_{2}$ does not belong since $h=0.05>h_{1}=0.0494$.

Suppose now that $\beta<0$ and consider the following possibilities for $\theta$.

4.2. Case $\theta=0$. Let $\theta=0$. Then

$$
f(h)=\frac{1}{2} \alpha \beta h^{2}+\alpha^{2} h+2 \alpha+\sigma^{2} .
$$

Since $2 \alpha+\sigma^{2}<0$ and $\alpha \beta>0$ then $f(h)<0$ for $h \in\left[0, h_{1}\right)$, where

$$
h_{1}=\frac{\sqrt{\alpha^{4}-2 \alpha \beta\left(2 \alpha+\sigma^{2}\right)}-\alpha^{2}}{\alpha \beta}>0 .
$$


For example, if $\alpha=-10, \beta=-1000, \sigma^{2}=1$ then $h_{1} \approx 0.0524$. Changing $\beta$ to $\beta=-1200$ we obtain $h_{1} \approx 0.0486<0.05$. On Figure 4.1 the point $B_{1}(-10,-1000)$ belongs to the stability region with $\theta=0$ and the point $B_{2}(-10,-1200)$ does not belong.

4.3. Case $\theta=1 / 2$. Let $\theta=1 / 2$. Then

$$
f(h)=-\frac{1}{2} \alpha \beta h^{2}+\left(\alpha^{2}-\beta\right) h+2 \alpha+\sigma^{2}
$$

Since

$$
D=\left(\alpha^{2}-\beta\right)^{2}+2 \alpha \beta\left(2 \alpha+\sigma^{2}\right)=\left(\alpha^{2}+\beta\right)^{2}+2 \alpha \beta \sigma^{2}>0
$$

then $f(h)<0$ for $h \in\left[0, h_{1}\right)$, where

$$
h_{1}=\frac{\alpha^{2}-\beta-\sqrt{D}}{\alpha \beta}>0 .
$$

For example, if $\alpha=-30, \beta=-1000, \sigma^{2}=1$ then $h_{1} \approx 0.0545$. Changing $\beta$ on $\beta=-1200$ we obtain $h_{1} \approx 0.0472$. On Figure 4.1 the point $C_{1}(-30,1000)$ belongs to the stability region with $\theta=1 / 2$ and the point $C_{2}(-30,-1200)$ does not belong to this region.

4.4. Case $\theta \in(1 / 2,1]$. Let $\theta \in(1 / 2,1]$. From (4.1) and (2.16) it follows that $f(h)<0$ for $h \leq 0$. So $f(h)<0$ for $h \in\left[0, h_{1}\right)$, where $h_{1}$ is the least root of the equation $f(h)=0$. For example, if $\alpha=-40, \beta=-1000, \sigma^{2}=1, \theta=0.75$ we obtain

$$
f(h)=187500 h^{3}-40000 h^{2}+3100 h-79=0
$$

and $h_{1} \approx 0.0511$. Changing $\beta$ to $\beta=-1200$ we obtain

$$
f(h)=270000 h^{3}-48000 h^{2}+3400 h-79=0
$$

with $h_{1} \approx 0.0431$. On Figure 4.1 the point $D_{1}(-40,-1000)$ belongs to the stability region with $\theta=3 / 4$ but the point $D_{2}(-40,-1200)$ does not belong to this region.

4.5. Case $\theta \in(0,1 / 2)$. Let $\theta \in(0,1 / 2)$. From (4.1) and (2.16) it follows that $f(0)<0$ and $(d f / d h)(0)>0$. It means that $f(h)<0$ for $h \in\left[0, h_{1}\right)$ where $h_{1}$ is the least positive root of the equation $f(h)=0$. For example, if $\alpha=-20, \beta=-1200, \sigma^{2}=1, \theta=1 / 4$ then

$$
f(h)=-90000 h^{3}+1000 h-39
$$

and $h_{1} \approx 0.0508$. Changing $\beta$ to $\beta=-1300$ we obtain

$$
f(h)=-105625 h^{3}+1050 h-39=0
$$

with $h_{1} \approx 0.0489$. On Figure 4.1 the point $E_{1}(-20,-1000)$ belongs to the stability region with $\theta=1 / 4$ but the point $E_{2}(-20,-1300)$ does not belong to this region. 


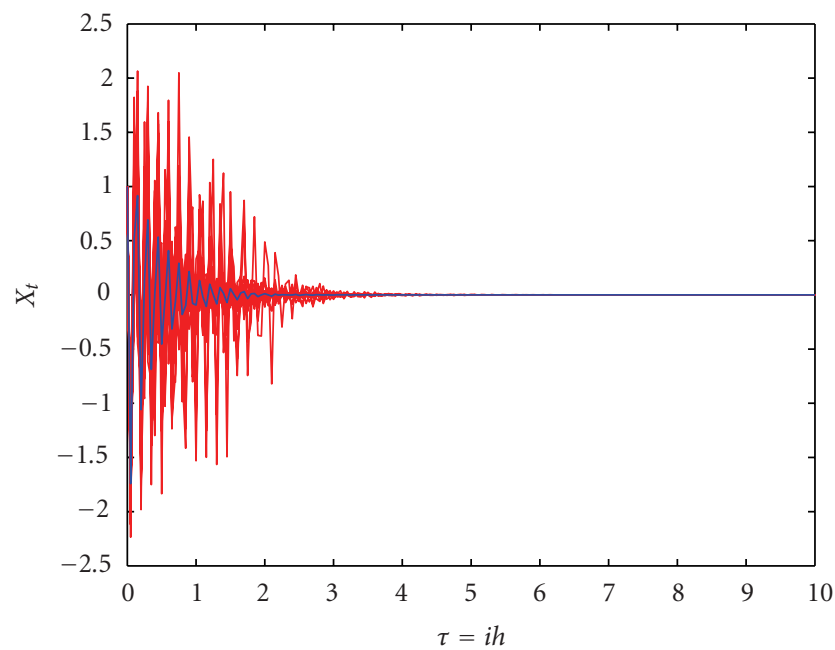

Figure 5.1. Trajectories of (3.1) with $m=0, \alpha=-55, \beta=-1000, \sigma^{2}=1, h=0.05, \theta=1, x_{0}=1$.

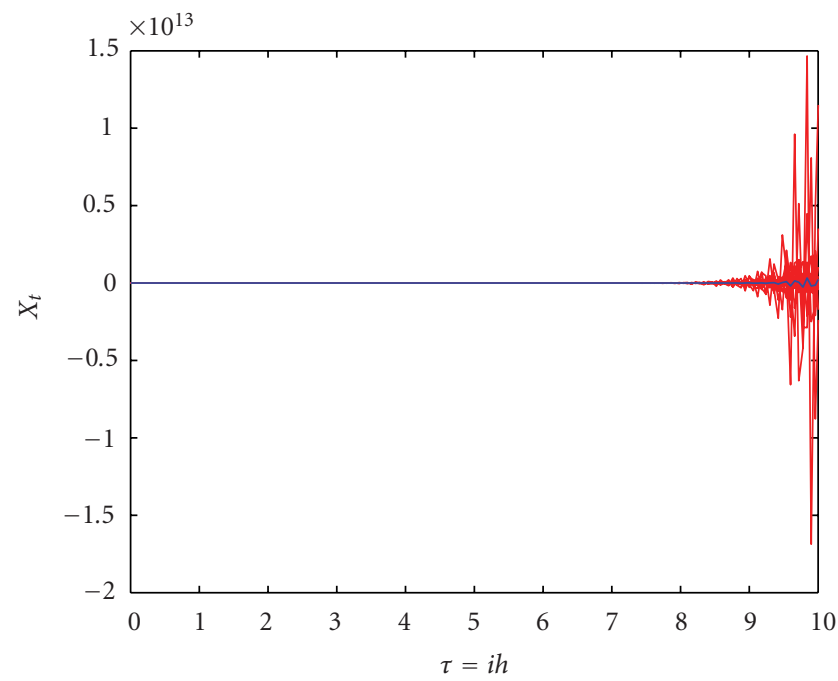

Figure 5.2. Trajectories of (3.1) with $m=0, \alpha=-55, \beta=-1000, \sigma^{2}=1, h=0.06, \theta=1, x_{0}=1$.

\section{Numerical experiments}

We illustrate some of our results with trajectories of (3.1). Note that in [30] an absolute correspondence of asymptotic mean square stability of the trivial solution and convergence of trajectories to zero was shown.

Figure 5.1 shows 50 trajectories of (3.1) with $m=0$ (i.e., without delay), $x_{0}=1, \alpha=$ $-55, \beta=-1000, \sigma^{2}=1, h=0.05, \theta=1$. The dark line represents the arithmetic mean of the trajectories, as it does for all the figures in this section. It is clear that we have a stable 


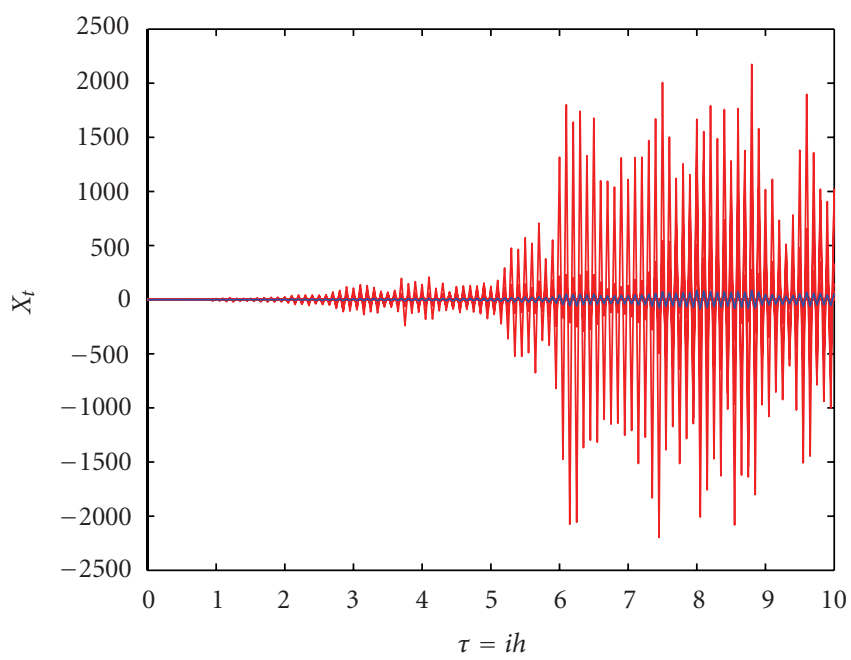

Figure 5.3. Trajectories of (3.1) with $m=0, \alpha=-40, \beta=-25, \sigma^{2}=1, h=0.05, \theta=0, x_{0}=1$.

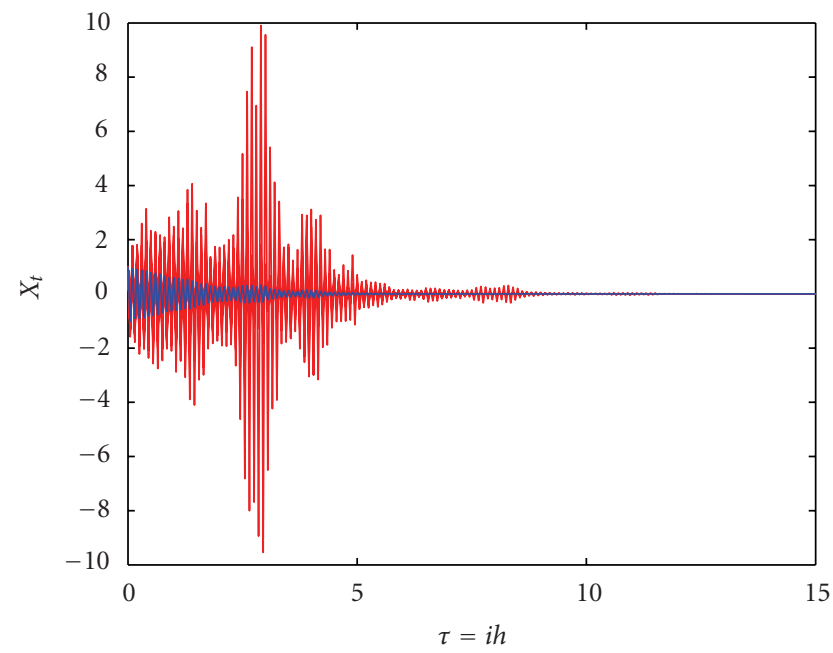

Figure 5.4. Trajectories of (3.1) with $m=0, \alpha=-40, \beta=-25, \sigma^{2}=1, h=0.05, \theta=1, x_{0}=1$.

system. If we change the parameter $h$ to $h=0.06$ we no longer have a stable system (as shown in Figure 5.2, as expected from examining Figure 3.5).

Figure 3.7 shows the regions of stability for different $\theta$ methods. We illustrate this point with Figures 5.3, 5.4 and 5.5. Each figure shows 50 trajectories with identical parameter values except for $\theta$. For Figure $5.3 \theta=0$, for Figure $5.4 \theta=1$, and for Figure $5.5 \theta=0.5$. The interesting point here is that for particular parameter values where the integrodifferential equation is asymptotically mean square stable we can choose a $\theta$ method 


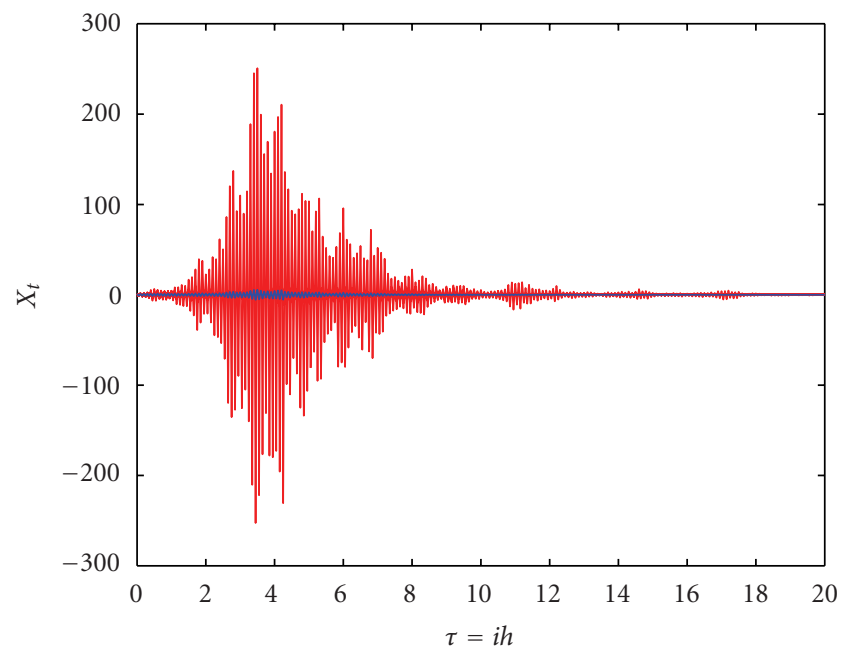

Figure 5.5. Trajectories of (3.1) with $m=0, \alpha=-40, \beta=-25, \sigma^{2}=1, h=0.05, \theta=0.5, x_{0}=1$.

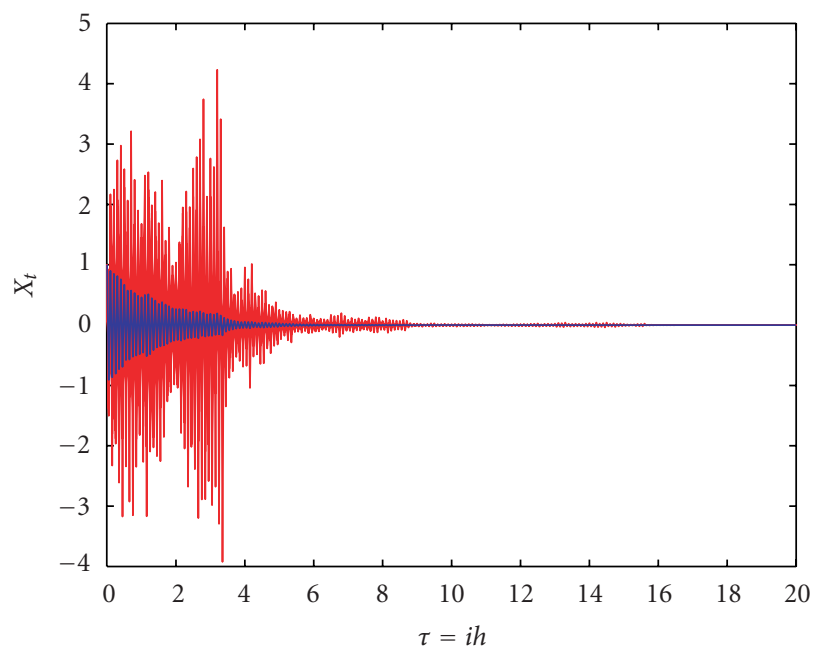

Figure 5.6. Trajectories of (3.1) with $m=0, \alpha=-39, \beta=-25, \sigma^{2}=1, h=0.05, \theta=0, x_{0}=1$.

which replicates this stability property. In Figure 5.3 the sufficient conditions for asymptotic mean square stability of the discrete system (i.e., $-38.8603<\alpha<-0.5147$, given the other parameters) are not satisfied and the trajectories are indeed unstable, whereas in Figures 5.4 and 5.5 the conditions (i.e., $-40.1103<\alpha<-1.7647$ for Figure 5.4 and $-39.4853<\alpha<-1.1397$ for Figure 5.5, given the other parameters) are satisfied and we have asymptotic mean-square stability. Figure 5.6 uses the same parameters as Figure 5.3 except that $\alpha=-39$. In this case the sufficient conditions are not satisfied for the discrete analogue (we are very close to satisfying them though) but we still have asymptotic mean 
square stability, thus verifying that are conditions are only sufficient and not necessary and sufficient. However we believe our experiments indicate that the sufficient conditions are very good ones.

\section{Acknowledgments}

This work has been completed with the financial assistance of NATO, grant reference PST.EV.979727, to whom the authors wish to express their thanks. We would also like to thank Dr John Edwards and Prof Neville Ford of University College Chester for helpful comments relating to early drafts of the work.

\section{References}

[1] V. N. Afanas'ev, V. B. Kolmanovskii, and V. R. Nosov, Mathematical Theory of Control Systems Design, Mathematics and Its Applications, vol. 341, Kluwer Academic, Dordrecht, 1996.

[2] H. Brunner and J. D. Lambert, Stability of numerical methods for Volterra integro-differential equations, Computing (Arch. Elektron. Rechnen) 12 (1974), no. 1, 75-89.

[3] H. Brunner and P. J. van der Houwen, The Numerical Solution of Volterra Equations, CWI Monographs, vol. 3, North-Holland, Amsterdam, 1986.

[4] S. Busenberg and K. L. Cooke, The effect of integral conditions in certain equations modelling epidemics and population growth, Journal of Mathematical Biology 10 (1980), no. 1, 13-32.

[5] A. Drozdov, Explicit stability conditions for stochastic integro-differential equations with nonselfadjoint operator coefficients, Stochastic Analysis and Applications 17 (1999), no. 1, 23-41.

[6] J. T. Edwards, N. J. Ford, and J. A. Roberts, The numerical simulation of the qualitative behaviour of Volterra integro-differential equations, Proceedings of Algorithms for Approximation IV (Huddersfield, 2001) (J. Levesley, I. J. Anderson, and J. C. Mason, eds.), University of Huddersfield, Huddersfield, 2002, pp. 86-93.

[7] J. T. Edwards, N. J. Ford, J. A. Roberts, and L. E. Shakhet, Stability of a discrete nonlinear integrodifferential equation of convolution type, Stability and Control: Theory and Applications. An International Journal 3 (2000), no. 1, 24-37.

[8] S. Elaydi and S. Sivasundaram, A unified approach to stability in integrodifferential equations via Liapunov functions, Journal of Mathematical Analysis and Applications 144 (1989), no. 2, 503531.

[9] N. J. Ford, C. T. H. Baker, and J. A. Roberts, Nonlinear Volterra integro-differential equationsstability and numerical stability of $\theta$-methods, Journal of Integral Equations and Applications $\mathbf{1 0}$ (1998), no. 4, 397-416.

[10] I. I. Gihman and A. V. Skorokhod, Stochastic Differential Equations, Izdat. Naukova Dumka, Kiev, 1968.

[11] J. Golec and S. Sathananthan, Sample path approximation for stochastic integro-differential equations, Stochastic Analysis and Applications 17 (1999), no. 4, 579-588.

[12] __ Strong approximations of stochastic integro-differential equations, Dynamics of Continuous, Discrete \& Impulsive Systems. Series B. Applications \& Algorithms 8 (2001), no. 1, 139151.

[13] D. J. Higham, X. R. Mao, and A. M. Stuart, Strong convergence of Euler-type methods for nonlinear stochastic differential equations, SIAM Journal on Numerical Analysis 40 (2002), no. 3, 10411063.

[14] _ Exponential mean-square stability of numerical solutions to stochastic differential equations, LMS Journal of Computation and Mathematics 6 (2003), 297-313.

[15] P. E. Kloeden and E. Platen, Numerical Solution of Stochastic Differential Equations, Applications of Mathematics (New York), vol. 23, Springer, Berlin, 1992. 
[16] V. B. Kolmanovskii and A. Myshkis, Applied Theory of Functional-Differential Equations, Mathematics and Its Applications (Soviet Series), vol. 85, Kluwer Academic, Dordrecht, 1992.

[17] V. B. Kolmanovskii and L. E. Shaikhet, A method for constructing Lyapunov functionals for stochastic systems with aftereffect, Differentsial'nye Uravneniya 29 (1993), no. 11, 1909-1920, 2022 (Russian), translation in Differential Equations 29 (1993), no. 11, 1657-1666 (1994).

[18] New results in stability theory for stochastic functional-differential equations (SFDEs) and their applications, Proceedings of Dynamic Systems and Applications, Vol. 1 (Atlanta, GA, 1993), Dynamic, Georgia, 1994, pp. 167-171.

[19] _ A method for constructing Lyapunov functionals for stochastic differential equations of neutral type, Differentsial'nye Uravneniya 31 (1995), no. 11, 1851-1857, 1941, translation in Differential Equations 31 (1995), no. 11, 1819-1825 (1996).

[20] - General method of Lyapunov functionals construction for stability investigation of stochastic difference equations, Dynamical Systems and Applications, World Sci. Ser. Appl. Anal., vol. 4, World Scientific, New Jersey, 1995, pp. 397-439.

[21] _ Construction of Lyapunov functionals for stochastic hereditary systems: a survey of some recent results, Mathematical and Computer Modelling 36 (2002), no. 6, 691-716.

[22] Some peculiarities of the general method of Lyapunov functionals construction, Applied Mathematics Letters. An International Journal of Rapid Publication 15 (2002), no. 3, 355-360.

[23] _ About one application of the general method of Lyapunov functionals construction, International Journal of Robust and Nonlinear Control 13 (2003), no. 9, 805-818, Special issue on Time-Delay Systems, RNC.

[24] J. D. Lambert, Numerical Methods for Ordinary Differential Systems: The Initial Value Problem, John Wiley \& Sons, Chichester, 1991.

[25] J. J. Levin and J. A. Nohel, Note on a nonlinear Volterra equation, Proceedings of the American Mathematical Society 14 (1963), 924-929.

[26] X. R. Mao, Stability of stochastic integro-differential equations, Stochastic Analysis and Applications 18 (2000), no. 6, 1005-1017.

[27] B. Øksendal, Stochastic Differential Equations: An Introduction with Applications, 5th ed., Universitext, Springer, Berlin, 1998.

[28] Y. Saito and T. Mitsui, Stability analysis of numerical schemes for stochastic differential equations, SIAM Journal on Numerical Analysis 33 (1996), no. 6, 2254-2267.

[29] L. E. Shaikhet, Necessary and sufficient conditions of asymptotic mean square stability for stochastic linear difference equations, Applied Mathematics Letters. An International Journal of Rapid Publication 10 (1997), no. 3, 111-115.

[30] _ Numerical simulation and stability of stochastic systems with Markovian switching, Neural, Parallel \& Scientific Computations 10 (2002), no. 2, 199-208.

[31] _ About Lyapunov functionals construction for difference equations with continuous time, Applied Mathematics Letters. An International Journal of Rapid Publication 17 (2004), no. 8, 985-991.

[32] Construction of Lyapunov functionals for stochastic difference equations with continuous time, Mathematics and Computers in Simulation 66 (2004), no. 6, 509-521.

[33] L_Lapunov functionals construction for stochastic difference second-kind Volterra equations with continuous time, Advances in Difference Equations 2004 (2004), no. 1, 67-91.

Leonid E. Shaikhet: Department of Higher Mathematics,

Donetsk State University of Management, Donetsk 83015, Ukraine

E-mail address: leonid.shaikhet@usa.net

Jason A. Roberts: Mathematics Department, University of Chester,

Chester CH14BJ, England

E-mail address: j.roberts@chester.ac.uk 